MacWilliam, I. C. (1959). J. gen. Microbiol. 21, 410-414

\title{
A Survey of the Antibiotic Powers of Yeasts
}

\author{
By I. C. MACWILLIAM \\ Brewing Industry Research Foundation, Nutfield, Surrey
}

\begin{abstract}
SUMMARY: Examination of the antibiotic powers towards bacteria and moulds of 150 strains of yeasts from the British National Collection of Yeast Cultures has revealed that whereas none exhibited marked antibacterial properties certain of them inhibited the growth of moulds. Strains of Candida pulcherrima, when grown on a malt + yeast + peptone + glucose agar medium inhibited the growth of strains of certain Mucor, Fusarium and Penicillium spp. The inhibition was due to pulcherriminic acid, a derivative of the red pigment, pulcherrimin, formed by Candida pulcherrima. It inhibited growth of the moulds completely at 5000 p.p.m. and partially at 500 p.p.m.
\end{abstract}

Only one systematic study has been reported in which the inhibitory powers, against bacteria and other organisms, of a large number of different strains of yeasts have been observed. In a brief report, Koch (1952) described the screening, by means of a diffusion plate method, of about 150 yeasts including distilling, baking, brewing and other strains against the bacteria, Bacillus subtilis, Serratia marcescens and strains of Pedicoccus isolated from beer. The yeasts Brettanomyces bruxellensis, Schizosaccharomyces pombé and brewing strains of Saccharomyces cerevisiae and $S$. carlsbergensis were found to inhibit the growth of $B$. subtilis and the pediococci but other species of yeast were found to produce substances which stimulated the growth of the bacteria. No attempt was made by Koch to isolate the substances producing inhibition of bacterial growth. Other workers have used only two or three strains of yeast but have carried out fractionations of the yeast cell constituents and culture fluids to concentrate the active materials. In a review of investigations made before 1949, Florey et al. (1949) noted that unsaturated fatty acids from brewers' and bakers' yeasts and from Debaryomyces mucosus and Torulopsis utilis, and succinic acid or other acidic substances from Torulopsis utilis var. major possess antibacterial properties. Later work has shown that cyclic peptides from bakers' yeast (Motzel \& Cook, 1958), certain proteins from brewers' and bakers' yeast (Parfentjev, 1955, 1957 $a, b$ ), the crystalline carotenoid lusomycin from Rhodotorula glutinis var. basitarica (Prista, 1952) and unidentified substances from $\boldsymbol{S}$. cerevisiae (Skorodumowa, 1954) all inhibit the growth of certain strains of bacteria.

The National Collection of Yeast Cultures provides a very wide selection of yeasts and the opportunity has been taken to survey the antibiotic properties of a representative number of these cultures. At least one strain of each species listed in the catalogue has been tested, by a diffusion plate method, for inhibitory action towards six selected bacteria and against certain moulds. In addition the effects of a number of the concentrated culture fluids from the yeasts on the growth of bacteria and moulds have been observed. 


\section{METHODS}

Organisms. 153 strains of yeast, comprising Brettanomyces, (5) Candida (31), Cryptococcus (5), Debaryomyces (4), Endomycopsis (3), Hansenula (7), Kloeckera (5), Nadsonia (2), Oospora (2), Pichia (6), Rhodotorula (10), Saccharomyces (44), Schizosaccharomyces (2), Sporobolomyces (5), Torulopsis (13), Trichosporon (3), and one each of Bullera, Hanseniaspora, Lipomyces, Saccharomycodes, Schwanniomyces and Trigonopsis were tested. Some of the bacteria were obtained from the National Collection of Type Cultures, (NCTC) Colindale, London, and the rest had been isolated in the Foundation. The cultures of moulds were kindly supplied by the Commonwealth Mycological Institute (CMI), Kew, England. Details are given in Table 1.

Table 1. Bacteria and moulds used as test organisms

(a) Bacteria

Staphylococcus aureus

Escherichia coli

Acetobacter sp.

Bacillus cereus

Flavobacterium proteus

Pseudomonas

aeruginosa

(b) Fungi

Rhizopus stolonifer

Fusarium oxysporum

F. equiseti

Penicillium periculosum

Mucor plumbeus

$\begin{array}{cccc}\text { Gram } & \text { Strain } & \begin{array}{c}\text { Growth } \\ \text { medium } \\ \text { used }\end{array} & \begin{array}{c}\text { Temperature } \\ \text { of } \\ \text { incubation }\end{array} \\ \text { reaction } & \text { No. } & \left(^{\circ}\right)\end{array}$

\begin{tabular}{|c|c|c|}
\hline+ & NCTC 6571 & $\begin{array}{l}\text { Nutrient broth } \\
\text { agar }\end{array}$ \\
\hline - & $*$ & $\begin{array}{l}\text { Nutrient broth } \\
\text { agar }\end{array}$ \\
\hline- & $*$ & $\begin{array}{l}\text { Acetobacter } \\
\text { medium }\end{array}$ \\
\hline+ & $*$ & $\begin{array}{l}\text { Nutrient broth } \\
\text { agar }\end{array}$ \\
\hline- & * & $\begin{array}{l}\text { Deep-liver } \\
\text { medium }\end{array}$ \\
\hline- & NCTC 6570 & $\begin{array}{l}\text { Nutrient broth } \\
\text { agar }\end{array}$ \\
\hline & CMI 17313 & MYPG† \\
\hline & CMI 53097 & MYPG† \\
\hline & CMI 49888 & MYPG $\dagger$ \\
\hline & CMI 61383 & MYPG $\dagger$ \\
\hline & CMI 74386 & MYPG† \\
\hline
\end{tabular}

* These cultures were isolated in the Foundation.

$\uparrow$ Malt + yeast + peptone + glucose agar.

Media. The bacteria and moulds were maintained and tested on the appropriate media listed in Table 1. Nutrient broth agar was prepared as described by Mackie \& McCartney (1949) and the Acetobacter, deep-liver and malt + yeast + peptone + glucose + agar (MYPG agar) media as described by Haynes, Wickerham \& Hesseltine (1955). The yeasts were grown on solid or liquid MYPG medium. The iron-rich medium for cultivation of Candida pulcherrima was prepared as described by Cook \& Slater (1954).

Materials tested. Pulcherriminic acid (3:6 di-isobutyl-4:5-dihydro-2:4dihydroxy-5-oxo-pyrazine-1-oxide) was isolated as described by Cook \& Slater (1956). Culture fluids (500 ml.) from the yeasts Brettanomyces bruxellensis (NCYC 362), Candida pulcherrima (166, 371, 372, 373), Candida utilis 
$(168,359)$, Saccharomyces carlsbergensis $(112,450)$, Saccharomyces cerevisiae (232, 240, 447), Schizosaccharomyces pombé (132), after 1 week's growth were separated from the organisms by centrifugation at $10,000 \mathrm{rev} . / \mathrm{min}$. and freeze-dried. The resulting solid was redissolved in sterile water $(5 \mathrm{ml}$.).

Methods of testing. Three methods were used for detecting growth inhibition.

1. Blocks of agar ( $1 \mathrm{~cm} .{ }^{2}$ and of the thickness of the medium) bearing a single yeast colony were cut with a sterile scalpel from $48 \mathrm{hr}$. cultures on MYPG agar and placed on the surface of a dry plate of suitable medium previously surface-inoculated with a saline suspension $(0.1 \mathrm{ml}$.) of the bacterium or mould under test. The medium was examined for zones of inhibition of growth around the blocks after aerobic incubation for 1-4 days at $25^{\circ}$ or $37^{\circ}$ (see Table 1).

2. The sensitivity of certain moulds was tested by streaking a loopful of a young yeast culture directly on to the surface of the appropriate medium previously inoculated with the mould as described above. The plates were then incubated and examined for zones of inhibition as previously described.

$3 a$. Freeze-dried culture fluids redissolved in one hundredth of their

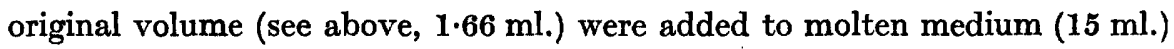
just before pouring plates. These were then surface-inoculated and incubated and the amount of growth compared visually with that obtained on the control medium devoid of culture fluid.

$3 b$. The inhibitory properties of pulcherriminic acid were tested similarly, concentrated solutions in $\mathbf{5 0} \%$ aqueous pyridine being used to give final concentrations in the medium of 5000, 500 and 50 p.p.m. For comparison, plates were prepared of medium containing the same amount of pyridine but no pulcherriminic acid.

\section{RESULTS}

Bacteria. In none of the tests in which bacteria were used as test organisms was their growth inhibited even slightly by substances secreted by the yeasts. In many cases the bacteria showed enhanced growth around the agar blocks containing the yeasts. Neither concentrated culture fluids from some of the yeasts nor pulcherriminic acid produced inhibition of growth.

Fungi. Most of the substances secreted by yeasts appeared also to enhance the growth of the moulds with the one exception, however, that strains of Candida pulcherrima, when grown in the presence of Fusarium, Penicillium and Mucor organisms produced zones of inhibition varying from 0.1 to $0.5 \mathrm{~cm}$. diam. around the yeast colony. The size of the zone in which growth of the test organism was prevented varied considerably from one batch of medium to another. The inhibition appeared to be inversely related to the amount of the red pigment, pulcherrimin, formed in the medium. Indeed, when an iron-rich medium (see above) was used and there was a very large production of pulcherrimin by the yeast no inhibition of mould growth was observed. After prolonged growth, the moulds completely overlapped the yeast colony on the latter medium, but did not do so on the normal medium where inhibition of 
growth was observed. Concentrations of 5000 p.p.m. pulcherriminic acid completely inhibited growth of Penicillium, Fusarium and Mucor spp. but did not inhibit Rhizopus stolonifer. Concentrations of 500 p.p.m. of the acid produced a retardation of growth, four days instead of two days being required for full growth. On the other hand culture fluids from Candida pulcherrima or from other yeasts produced no significant inhibition of growth. Efforts were made to extract pulcherriminic acid from the freeze-dried culture fluids from C. pulcherrima by using pyridine, in which the acid is readily soluble and which was itself without action on the mould. However, only very small quantities of material were obtained which did not inhibit or retard growth of the moulds. All four strains of C. pulcherrima from the National Collection (Nos. 166, 371, 372, 373) produced inhibition but strain 373 produced the greatest effect followed by nos. 372, 371, and 166 in descending order of activity. Incidentally, strain no. $\mathbf{3 7 3}$ produced the greatest yield of pulcherrimin on the iron-rich medium.

\section{DISCUSSION}

The aim of the present study was to survey rapidly a wide range of yeasts with a view to detecting the production of antibiotic substances of potency comparable to that of the antibiotics at present commercially available; the range of conditions of testing was necessarily restricted. Consequently, substances having weak inhibitory powers or which diffused slowly into the agar, may have gone undetected; this possibly explains the discrepancies between the present results and those of Koch (1952) who reported the presence in brewing and other yeasts of substances which inhibited bacterial growth and brought about lysis of test organisms. Nevertheless, it is clear that the liberal production of antibiotic substances of potencies comparable to those of the commercial antibiotics would have been detected by the methods used. It is concluded that yeasts are not a promising source of antibiotics as compared with certain other organisms.

The inhibition of moulds by pulcherriminic acid is perhaps not unexpected since the compound has been shown to possess structural similarities to the antibiotic aspergillic acid (N-hydroxy-2-oxo-3:6-dibutylpyrazine; White, 1940; Dutcher \& Wintersteiner, 1944; Dunn, Gallacher, Newbold \& Spring, 1949). From the present results it seems reasonable to presume that in the liquid and iron-rich media most of the pulcherriminic acid which was formed by the yeast was converted, by combination with ferric ion, to pulcherrimin which, being almost completely insoluble in water and organic solvents, did not inhibit growth. In the solid medium, exhaustion of ferric ion in places of yeast growth probably occurred and some pulcherriminic acid was present to produce inhibition of mould growth.

On the basis of the results shown above and of recent findings, a study of the antibiotic properties of other oxo-pyrazines of greater solubility in water than aspergillic acid and pulcherriminic acid may well yield rewarding results.

The author wishes to thank Dr A. H. Cook, F.R.S., for help and guidance during the course of this work. 


\section{REFERENCES}

Cook, A. H. \& Slater, C. A. (1954). Metabolism of 'wild' yeasts. 1. The chemical nature of pulcherrimin. J. Inst. Brewing, 60, 213.

Coor, A. H. \& Stater, C. A. (1956). The structure of pulcherrimin. J. chem. Soc. p. 4133.

Dunn, G., Gallagher, J. J., Newbold, G. T. \& Spring, F. S. (1949). Aspergillic acid. Part 1. J. chem. Soc. S126.

Dutcher, J. D. \& WiNTERSteINER, O. (1944). The structure of aspergillic acid. J. biol. Chem. 155, 359.

Florey, H. W., Chain, E., Heatley, N. G., Jennings, M.A., Sanders, A. G., Abraham, E. P. \& Florey, M. E. (1949). The Antibiotics, Vol. 1, p. 349. Oxford: Oxford Medical Publications.

Haynes, W. C., Wickerham, L. J. \& Hesseltine, C. W. (1955). Maintenance of cultures of industrially important organisms. J. appl. Microbiol. 3, 361.

Koch, R. (1952). Antibiotic action of yeasts on bacteria. Die Brauerei, 6, 307.

Mackie, T. J. \& McCartiney, J. E. (1949). Handbook of Practical Bacteriology, 8th edn. p. 144. Edinburgh: E. and S. Livingstone Ltd.

MotzeL, W. \& Cook, E. S. (1958). Antibiotic substances from yeast. Nature, Lond. 182, 455.

Parfentuev, I. A. (1955). Nonspecific desensitization against $H$. pertussis hypersensitivity by injection of yeast protein. Fed. Proc. 14, 474.

Parfentuev, I. A. (1957 a). Yeast protein with antibiotic properties. Fed. Proc. 16, 428.

Parfentuev, I. A. (1957b). Prenatal protection of mice by yeast antibiotic (malucidin). Science, 126, 928.

Prista, L. N. (1952). Lusomycin; the pigments of Rhodotorula glutinis. Congr. Luso-Espan. farm., 11 Congr., 2, 1 a Sec., 274 in Chem. Abstr. (1954), 48, 13,807 a.

Skorodumowa, A. M. (1954). Antibiotic properties of carbohydrate-fermenting yeasts. Microbiology, Moscow, 23, 419.

Whiтe, E. C. (1940). Bactericidal filtrates from a mold culture. Science, 92, 127.

(Received 3 April 1959) 